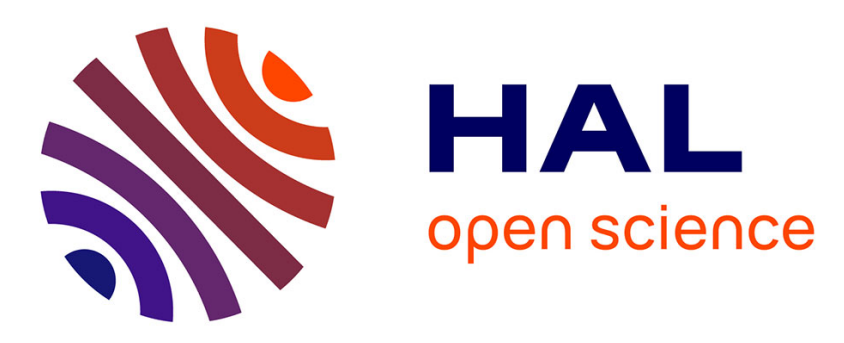

\title{
Application of stereovision to the mechanical characterisation of ceramic refractories reinforced with metallic fibres
}

Jean-José Orteu, Thierry Cutard, David Garcia, Emmanuel Cailleux, Laurent Robert

\section{To cite this version:}

Jean-José Orteu, Thierry Cutard, David Garcia, Emmanuel Cailleux, Laurent Robert. Application of stereovision to the mechanical characterisation of ceramic refractories reinforced with metallic fibres. Strain, 2007, 43 (2), pp.96-108. 10.1111/j.1475-1305.2007.00322.x . hal-01644896

\author{
HAL Id: hal-01644896 \\ https://hal.science/hal-01644896
}

Submitted on 21 Feb 2018

HAL is a multi-disciplinary open access archive for the deposit and dissemination of scientific research documents, whether they are published or not. The documents may come from teaching and research institutions in France or abroad, or from public or private research centers.
L'archive ouverte pluridisciplinaire HAL, est destinée au dépôt et à la diffusion de documents scientifiques de niveau recherche, publiés ou non, émanant des établissements d'enseignement et de recherche français ou étrangers, des laboratoires publics ou privés. 


\title{
Application of Stereovision to the Mechanical Characterisation of Ceramic Refractories Reinforced with Metallic Fibres
}

\author{
J. J. Orteu, T. Cutard, D. Garcia, E. Cailleux and L. Robert \\ École des Mines d'Albi Carmaux, Campus Jarlard, 81013 Albi, France
}

\begin{abstract}
Optical methods that yield displacement or strain fields are now emerging significantly in the mechanical sciences. At the Research Center on Tools, Materials and Forming Processes (CROMeP) at École des Mines d'Albi, a binocular stereovision system has been developed that can be used to measure: (a) the three-dimensional (3D) shape of a static object, or (b) the surface strains of an object undergoing some 3D mechanical or thermal stress. In this paper, the application of the stereovision technique to investigate the behaviour of ceramic refractories reinforced with metallic fibres is presented: (i) after the rupture of the sample for assessing the 3D orientation of the fibres in order to correlate a micro-mechanical model of fibre pullout with the macro-mechanical results of tensile tests, and (ii) during a tensile test for measuring the 3D displacement/strain field around a notch.
\end{abstract}

KEY WORDS: ceramic refractories, digital image correlation (DIC), experimental mechanics, metallic fibres, stereovision, stereo correlation, 3D metrology

\section{Introduction}

Optical methods that yield displacement or strain fields are now emerging significantly in the mechanical sciences. Much research has been done on two dimensional (2D) displacement/strain meas urement from a single camera (see Refs $[1,2]$ for a review). The proposed methods yield only in plane strains and lead to some experimental difficulties when ensuring the relative position of camera sensor and sample planes. To circumvent these limitations, some studies have been performed on three dimen sional (3D) displacement/strain measurement using two cameras or a single moving camera [3 9].

In the Research Center on Tools, Materials and Forming Processes (CROMeP) at École des Mines d'Albi, non contact methods for mechanical and thermal measurements in the field of material pro cessing, for the study of the behaviour of materials and structures, and for process monitoring were developed in the Artificial Vision and Thermography laboratory. In particular, a binocular correlation based stereovision technique has been developed that can be used to measure:

(a) the 3D shape of a static object, or

(b) the strain of an object undergoing 3D mechan ical or thermal stress.
Studies have focused on the strong ${ }^{1}$ and accurate cal ibration of cameras or binocular stereovision sensors $[11,12]$, the measurement of $3 \mathrm{D}$ shapes by stereovision (in particular by stereo correlation), the measurement of 3D displacement fields/surface strains by using ste reo correlation and pixel tracking [by digital image correlation (DIC)] in conjunction in a sequence of stereo images. As a result of the metrological aspect of the study, particular attention was given to ensure the accuracy of the proposed methods (accuracy of the camera calibration, accuracy of the image matching, subpixel correlation, etc.). The stereovision technique has been used to measure 3D strains on stamped sheet metal parts, on inflated elastomer membranes, and on metallic parts under heat treatment [12 16$]$.

In this paper, the application of the stereovision technique to study the mechanical behaviour of ceramic refractories reinforced with metallic fibres is presented:

\footnotetext{
As opposed to weak calibration [10]. Strong camera calibration techniques produce the most accurate calibration. They consist of imaging a special-purpose reference calibration target, whose structure is known a priori. Weak calibration techniques do not rely on the use of a calibration target. In the weakly calibrated case, point correspondences between stereo-images are the only information available.
} 
1 After the sample rupture: to measure the 3D orientation of the fibres in order to correlate a micro mechanical model of fibre pullout with the macro mechanical results of tensile tests.

2 During a tensile test: to measure the 3D displace ment/strain field around a notch.

In succeeding section, the improved stereovision technique for 3D shape and 3D displacement/strain measurement is presented. In the section following that, the application to ceramic refractories rein forced with metallic fibres is described. In the section 'Measurement of the 3D Orientation of Metallic Fibres', the application of stereovision to the 3D reconstruction of metallic fibres is presented. In the section following that, the application of stereo cor relation to the $3 \mathrm{D}$ displacement measurement during a tensile test on a pre notched sample is presented.

\section{Stereovision for 3D Shape and 3D Displacement/Strain Measurement}

Binocular stereovision is a technique for recovering the 3D structure of a scene from two different view points [see Figure 1 where $P(X, Y, Z)$ is the $3 \mathrm{D}$ point to be measured, $p_{1}\left(u_{1}, v_{1}\right)$ and $p_{2}\left(u_{2}, v_{2}\right)$ are its stereo projections in the images, $C_{1}$ and $C_{2}$ are the optical centres of the two cameras (Figure 2)]. From a pair of images, it is possible to compute the $3 \mathrm{D}$ coordinates of a physical 3D point by triangulation, assuming that:

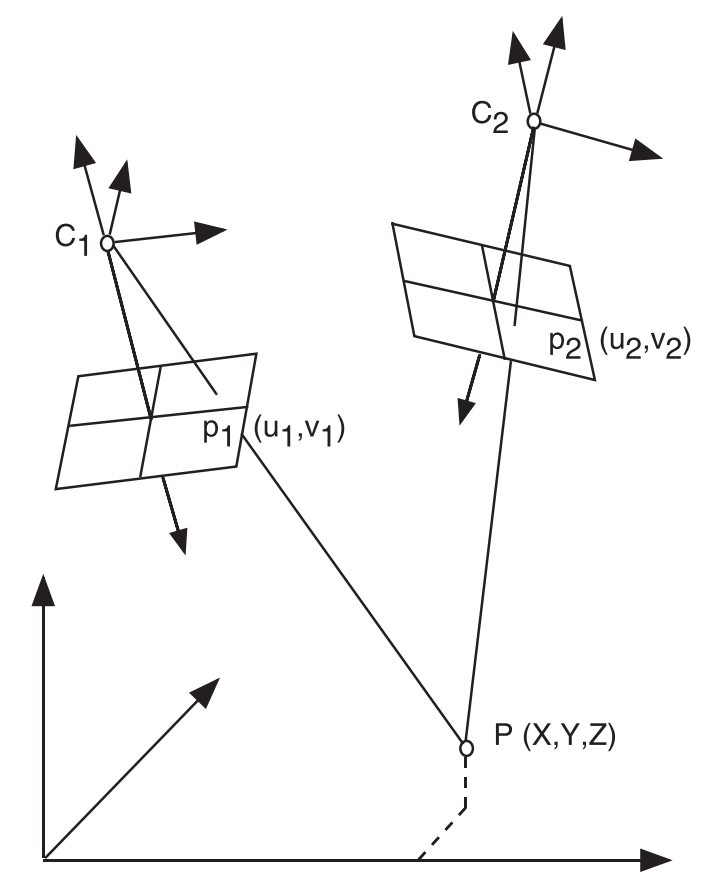

Figure I: Binocular stereovision

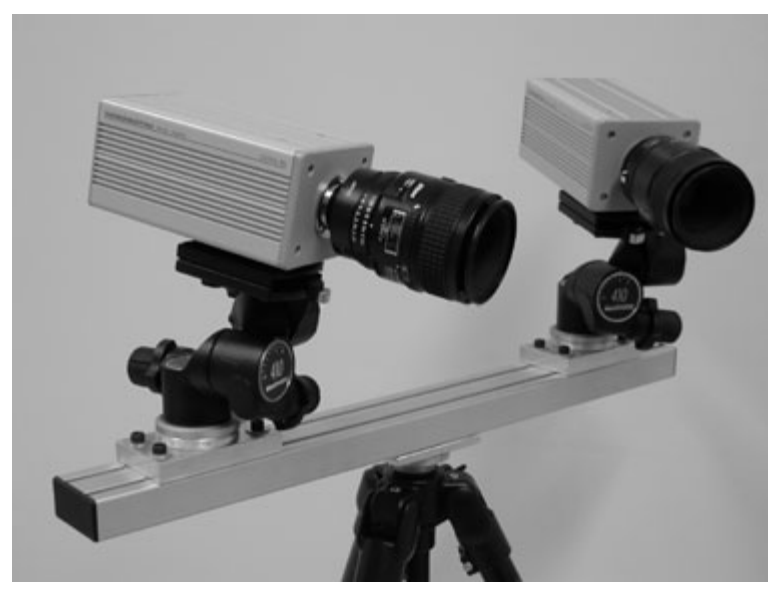

Figure 2: The stereo-rig used in our laboratory

1 The geometry of the stereo rig (i.e. the relative position and orientation of the two cameras) is known. This problem is solved by an off line camera calibration procedure.

2 The two image points $p_{1}$ and $p_{2}$ are matched, i.e. identified as corresponding to the same physi cal point $P$. This is called the stereo matching problem.

\section{Calibration of the stereovision sensor}

Camera calibration is an important task in 3D com puter vision, particularly when metric data are required for applications involving accurate dimen sional measurements. Calibrating a camera involves determining its intrinsic parameters and its position and orientation with respect to an arbitrary world reference frame [17]. Calibrating a stereovision sensor made up of two cameras involves determining the intrinsic parameters of each camera and the relative position and orientation between the two cameras. These calibration data are required to compute, by triangulation, the $3 \mathrm{D}$ coordinates of a point corres ponding to matched pixels on the two images.

An original and flexible technique has been devel oped to easily calibrate a camera or a stereovision sensor. Lens distortion is taken into account. The technique only requires the camera to observe a (pla nar) pattern shown at a few different orientations (typically between 10 and 20). The motion of the pattern need not be known and the pattern itself can be imprecise (i.e. its geometry need not be known accurately). Using a photogrammetric bundle adjust ment approach [18], the intrinsic parameters of each camera (including the distortion parameters), the 3D points of the pattern and the relative position and orientation between the two cameras are estimated all together. The technique is easy to use, flexible and leads to a highly accurate calibration $[11,12]$. 
The calibration parameters will be used throughout this paper at different stages for:

- the rectification of the pairs of stereo images;

- the correction of lens distortion;

- the calculation of the 3D position of a scene point from its stereo projections by triangulation.

\section{Rectification of the pair of stereo-images}

Given a point $p_{1}$ in image 1 , its corresponding point $p_{2}$ in image 2, termed 'stereo correspondent' (this is the stereo matching problem discussed in the section 'Stereo matching') appears to be always lying along a line of image 2 defined entirely by the coordinates of $p_{1}$, the relative position/orientation of the two cam eras and their intrinsic parameters: this is the so called epipolar line associated with $p_{1}[19,20]$ (see Figure 3 ). This geometric constraint inherent to any stereo imaging system, termed 'epipolar constraint', is very interesting because it simplifies the search for the stereo correspondent of a given point from a 2D search across the entire image to a $1 \mathrm{D}$ search along its epipolar line.

In the particular case where the cameras are per fectly aligned, the epipolar lines are parallel to the image rows (see Figure 4): this ideal configuration simplifies the search for correspondence because corresponding pixels are on the same row in both images. In this configuration, the difference between the column coordinates of two matched points is called 'disparity'.

In practice, the mechanical alignment of the cam eras is very difficult to obtain. Nevertheless, the prob lem can be solved at the processing level by applying a transformation (plane to plane homography) ${ }^{2}$ to each image of the initial stereo pair to obtain a new pair of stereo images corresponding to a virtual stereo rig with perfectly aligned cameras $[19,20]$. This rectifica tion procedure uses the calibration parameters com puted in the off line camera calibration phase. Notice that during the rectification, the real distorted images (because of optical lens distortion) are also trans formed into ideal distortion free images.

The image rectification procedure yields a pair of images that corresponds to an ideal stereo rig of dis tortion free and perfectly aligned cameras. This greatly simplifies the stereo matching phase. It should be noted that, in practice, neither the rectified images nor the undistorted images are computed. In fact, the computed rectification homographies and the distor

\footnotetext{
${ }^{2} \mathrm{~A}$ plane-to-plane homography is a bijective projective transformation mapping pairs of points between planes $[20,21]$.
}

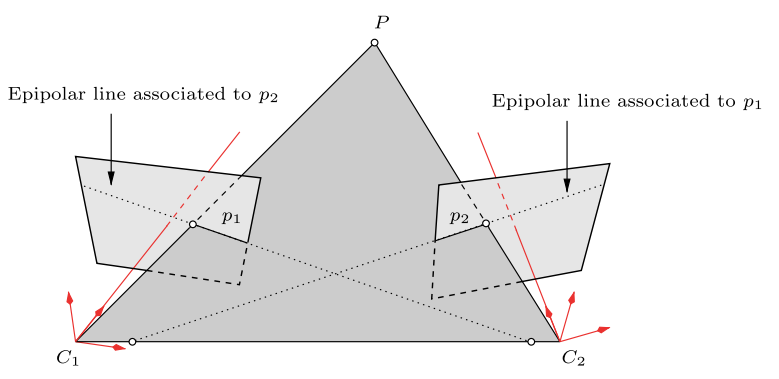

Figure 3: The camera optical centres $C_{1}$ and $C_{2}$, the $3 \mathrm{D}$ point $P$ and its images $p_{1}$ and $p_{2}$ lie in a common plane. This plane intersects each image plane in an epipolar line

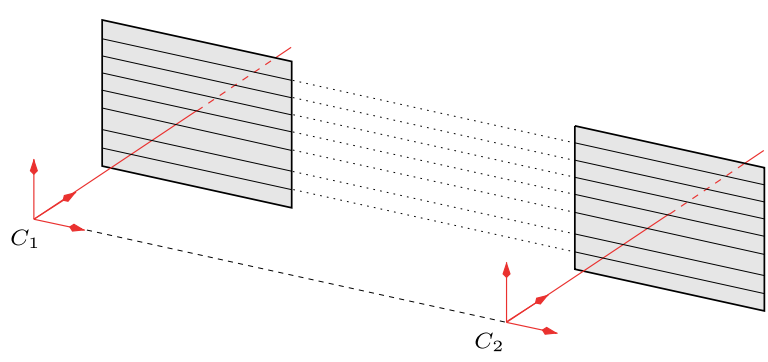

Figure 4: A rectified stereovision sensor: the epipolar lines are parallel to the image rows

tion parameters are used directly in the expression of the correlation function to work on the raw images. This new approach can achieve the highest matching accuracy by avoiding the pixel interpolations involved in the distortion correction and rectification of the images (see Refs [12, 16] for more details).

\section{Stereo-matching}

The main difficulty in stereovision is to establish cor respondences between pairs of images. Over the years, numerous algorithms for image matching have been proposed. They can be classified in two categories:

1 Feature matching: The algorithms first extract sali ent primitives from the images, such as edge seg ments or contours, and match them in the views being considered. These methods are fast because only a small subset of the image pixels is used, but usually give few matches (sparse disparity maps).

2 Template matching: The algorithms attempt to cor relate the grey levels of image patches in the views being considered, assuming that they present some similarity. The underlying assumption appears to be valid for relatively textured areas and for image pairs with small differences. These methods can give dense disparity maps.

In Ref. [7], a method based on stereovision for measuring strains in stamped 3D sheet metal parts was proposed. This method falls into category 1 as it requires that a predefined pattern (a grid of squares) 
be applied to the sheet surface before stamping. Each image of the stereo pair is processed independently in order to locate the grid intersections. The grid intersections are extracted in such a way as to allow an automatic matching between the two images, provided that the operator matches manually a single pair of points.

A stereo correlation technique that falls into cate gory 2 has also been developed (and is presented in this paper). With this technique, a regular grid need not be applied to the object and the meshes used to compute the local strains are generated at the post processing level (meshes of any size can be generated, and in particular small ones to take into account large strain gradients).

Correlation scores are computed by measuring the similarity of a fixed window in the first image to a shifting window in the second (see Figure 5). The second window is moved in the second image by integer increments along the corresponding image row (remember that a 'rectified space' is being worked with) and a curve of correlation scores is generated (see Figure 6). The correct matching corresponds to the highest peak provided that this peak is greater than a threshold $\left(S_{\min }\right)$. Note that a match is not accepted if the highest and the second highest peaks are within a minimum range $(\Delta)$. By definition, cor responding points have coordinates $(u, v)$ and $(u$, $v+d$ ) in left and right rectified images, and $d$, the distance in pixels, is the disparity (see Figure 5).

Figure 5 presents a gross simplification of stereo matching process: matching windows are assumed to be square and of the same size. In practice, because of local surface non planarity as well as projective dis tortion, a square window is most probably matched with a non square one as shown in Figure 7. So, we calculate the correlation between the fixed left win dow (a rectangle) and the shifting right window (a distorted rectangle). The optimal shape of the corre lation window in the right image is computed according to the local orientation and curvature of

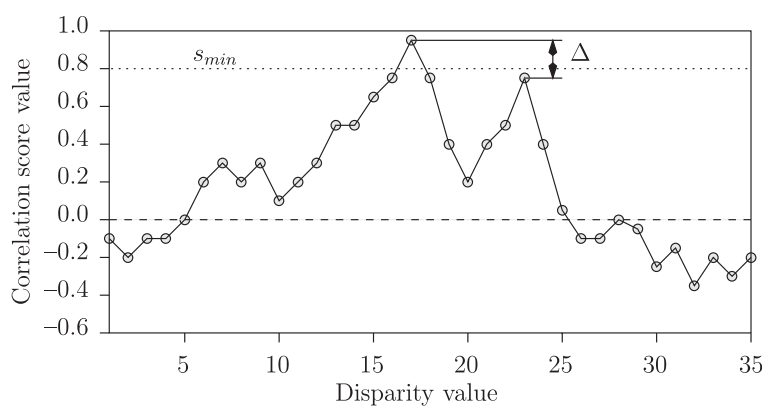

Figure 6: Curve of correlation scores

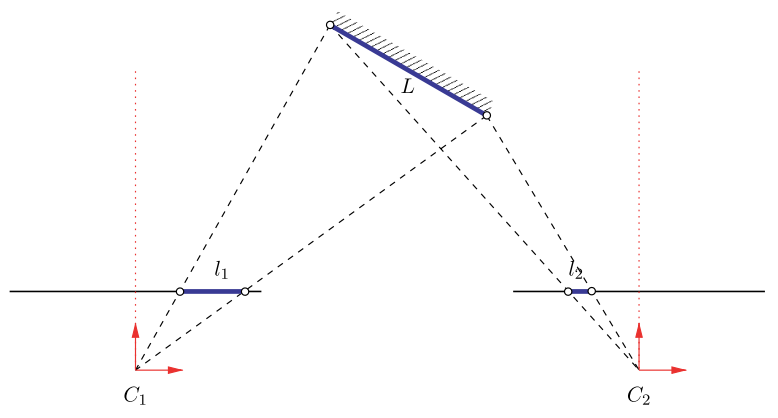

Figure 7: Projective deformation: the segment of length $L$ in space is projected to a segment of length $l_{1}$ in the left image and to a segment of length $l_{2} \neq l_{1}$ in the right image

the surface. Note that in Figure 6, the disparity is an integer value but in reality a subpixel correlation based stereo matching algorithm has been developed so the disparity is a real value. For more details, see Ref. 12, 16 .

As mentioned above, it is well known that the correlation technique is efficient on textured objects. When an object does not present a suitable random, contrasted texture, one may apply a thin coating of high contrast particles such as from spray painting [22], toner powder, ink, lithography, etc.

\section{D reconstruction}

Using the calibration parameters of each camera and the rectifying homographies, a classical triangulation method [23] can be used to compute the 3D position
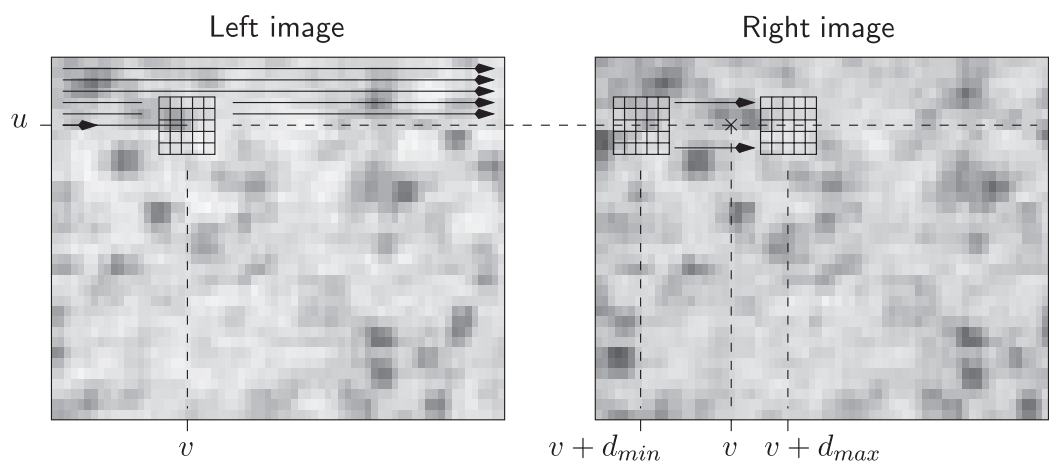

Figure 5: Correlation-based stereo-matching 
of a scene point corresponding to a stereo pair of image points (see Figure 1), assuming that their distortion have been corrected. By repeating this operation for a large number of stereo matched pairs, we can obtain the 3D shape of an object.

\section{D displacement measurement}

Using the stereovision technique, the shape variation of an object can be measured by analysing a sequence of pair of stereo images. Nevertheless, in experimen tal mechanics, we are generally interested in the surface strain field, that can be obtained by tracking the displacement of some points at the surface of an object undergoing some mechanical or thermal stress.

As already mentioned, much research has been done on 2D displacement/strain measurement, using a single camera, on gridded objects or using correla tion based techniques. These methods can give only the in plane strains. The stereovision technique allows measurement of the 3D displacement field of an object undergoing 3D deformation.

From the pair of stereo images taken at time $t_{0}$, the $3 \mathrm{D}$ shape of the object at time $t_{0}$ can be computed. From the pair of stereo images taken at time $t_{1}$, the $3 \mathrm{D}$ shape of the object at time $t_{1}$ can be computed. In addition, by matching the two images taken by the left camera (or the right one) at time $t_{0}$ and $t_{1}$ (this is called temporal matching or tracking), the $3 \mathrm{D}$ dis placement corresponding to each image point can be computed (see Figure 8).

The temporal matching problem is similar to the classical 2D displacement measurement problem. It is important to note that, in this case, the epipolar constraint cannot be used because two time varying images taken by a single camera are being dealt with. Depending on whether the object is marked with a grid or not the temporal matching problem can be tackled using feature matching techniques or a cor relation based algorithm. When 3D displacement fields are measured by stereo correlation, stereo cor relation (i.e. stereovision + correlation based stereo

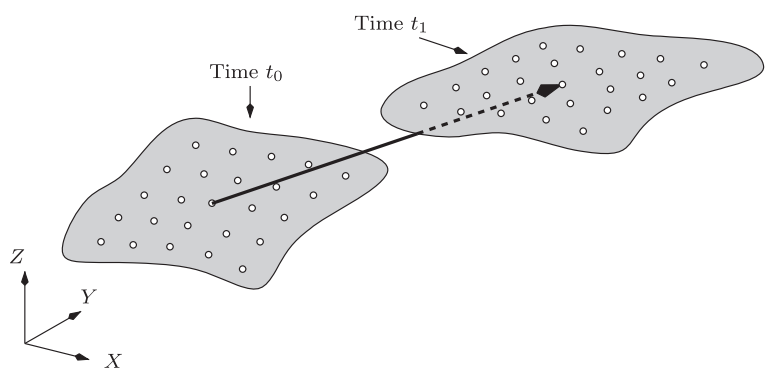

Figure 8: $3 \mathrm{D}$ displacement field computation matching) that gives 3D information and pixel tracking by correlation (DIC) in the sequence of ste reo images are used in conjunction. In order to achieve better accuracy, an improved method (com bining temporal tracking and stereo correlation) is proposed that operates simultaneously on the two pairs of stereo images [16].

When the object to be measured can be observed during its deformations (see the section '3D Dis placement Field Measurement' for an example), a sequence of time varying pairs of stereo images can be acquired with a sufficiently fast acquisition rate to guarantee a small deformation between each image of the sequence. However, in the case of sheet metal forming, generally the part cannot be observed dur ing its deformation. Only a pair of images of the undeformed blank and a pair of images of the deformed part are available. The stereo correlation technique has been applied successfully to deformed parts with up to $30 \%$ of strain between two successive pairs of stereo images [16].

\section{$3 D$ strain computation}

In Ref. [7], the strain field was computed by analysing the initial and final configuration of an array of points marked on the surface. Each square element of the grid pattern was divided into triangular sub elements. The principal strain values in each element were determined by comparing the undeformed and deformed triangles. In the present study, no regular grid is marked on the surface of the object but we can compute the 3D mesh resulting from the 3D recon struction of a Delaunay triangular mesh defined in an image (sparse reconstruction; see Figure 9). The strain field can be computed by using each 3D triangular element of the meshes before and after the defor mation. A finite element software can be used for these computations. In our laboratory, the software Abaqus $^{\mathrm{TM}}$ is often used for these computations. A 3D triangular mesh is generated from the $3 \mathrm{D}$ points and Abaqus $^{\mathrm{TM}}$ (www.abaqus.com) computes the strain values from the $3 \mathrm{D}$ displacements imposed at the nodes of the mesh [24].

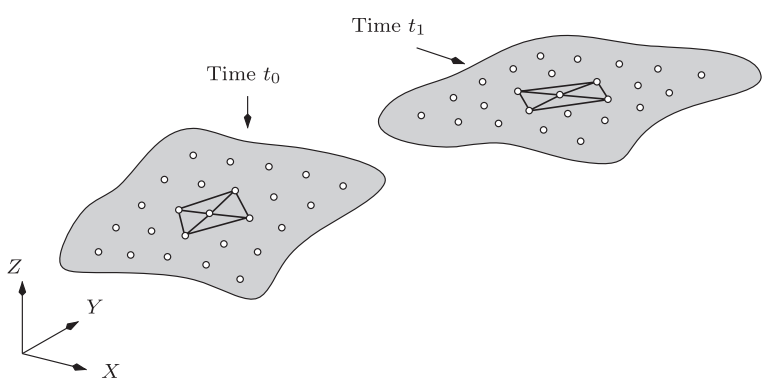

Figure 9: 3D strain field computation 


\section{Accuracy}

The accuracy of 3D reconstruction depends on many factors:

- the quality of the cameras and their resolution;

- the configuration of the two cameras (angle between their optical axis) which governs the triangulation accuracy;

- the accuracy of the stereovision sensor calibration;

- the accuracy of the matched features in the images.

The accuracy of the matched features depends on the type of extracted feature. In Ref. [7] gridded objects were used and the grid intersection extraction accu racy was $1 / 30$ pixel. In Ref. [12] it has been shown that the DIC technique leads to an accuracy better than $1 / 100$ pixel.

Using high resolution digital cameras with $1024 \times$ 1024 pixels each, the 3D reconstruction relative accuracy in object space (the accuracy of coordinate determination divided by the size of the object) achieved using stereo correlation under controlled laboratory conditions is about 1/50 000 (see Ref. [12] for a more detailed discussion on $3 \mathrm{D}$ reconstruction accuracy).

The strain values are computed by numerical deri vation from the displacements measured at several points distributed over a mesh. The strain at each point of the mesh is computed using neigh bouring points (see Figure 9). The accuracy of the strain computation depends on many factors: the discrete derivation scheme used (shape of the integ ration domain) [25], the accuracy of the displace ment measurement, and the computation basis (mesh element spacing). The method proposed can reach an accuracy of $0.05 \%$ (500 $\mu$ strain) on the computed strains, with triangular elements measur ing 50 pixels.

For example, for 2D DIC, using the integration domain shown in Figure 9 (four neighbouring points) and the discrete derivation scheme described Ref. 25 , the uncertainty for a 2D strain computation can be evaluated using the following relation:

$\Delta \varepsilon=\frac{\Delta d}{\sqrt{ } 2 \Delta x}$

where $\Delta \varepsilon$ is the uncertainty on strain computation, $\Delta d$ the uncertainty on displacement measurement and $\Delta x$ the computation basis for the discrete deri vation.

For example, with $\Delta d$ equal to $1 / 100$ pixel and a derivation basis $\Delta x$ equal to 14 pixels, the uncertainty on strain measurement $\Delta \varepsilon$ is equal to $0.05 \%$. An uncertainty 10 times lower $(0.005 \%)$ can be achieved by taking a derivation basis 10 times larger (140 pixels) but the result has much less local significance, which can be a problem with heterogeneous materials such those that are the object of the studies in this paper.

\section{Ceramic Refractories Reinforced with Metallic Fibres}

As cementitious materials, ceramic refractories are characterised by a quasi brittle macro mechanical behaviour [26, 27]. Incorporation of metallic fibres can significantly improve their mechanical perform ances in terms of toughness, strain capacity or energy absorption capacity. In such composites, fibres act as bridging ligaments behind the crack tip resisting crack propagation. Moreover, in practice the most rein forced concretes use short fibres with a quasi random distribution in the composite. Fibres at any angle can therefore intersect cracks [28]. Fibres can play an important role in crack initiation too. Before crack initiation, ceramic refractories often exhibit a non linear mechanical behaviour. This is due to damage processes that occur in these materials by micro cracking. An extension of the damage domain can be obtained with fibre reinforcement, which modifies or delays strain localisation mechanisms. Compared with civil engineering concretes, ceramic refractories have to sustain both mechanical loading and high temperatures and/or severe temperature gradients.

Ceramic refractories are heterogeneous materials. Their microstructures mainly consist of a cement based matrix, aggregates, porosity, microcracks and decohesions. Adding short metallic fibres creates a new degree of heterogeneity in these materials. This is of major importance when considering high tem perature microstructural behaviour. When varying the temperature, fibre/concrete interfaces will be the cites of large evolutions too [29]. As detailed hereafter, these evolutions are mainly related to damage. Dur ing the first thermal cycle, large stress levels appear near the fibre/concrete interface and are due to the thermal expansion mismatch between the two com ponents. As a result of such stress fields, a damage process is initiated in terms of radial matrix cracking and fibre/matrix decohesion. This latter mechanism occurs during the cooling of the first thermal cycle and at a transition temperature, which depends on the maximum temperature of the cycle. It leads to a decrease of the fibre pullout load level at room tem perature. The reversibility of this decohesion process leads to an important increase of the pullout per formance during high temperature tests. This is mainly due to the development of a fretting pressure 
at the fibre/matrix interface. Such a behaviour is of great interest for high temperature applications of composites based on these two components. This is particularly true to limit the brittle behaviour that characterises unreinforced ceramic refractories.

Two main questions have to be answered to design industrial parts based on short metallic fibre rein forced ceramic refractories. One deals with the opti misation of the composite design or formulation for a better high temperature mechanical behaviour. Some adjustable parameters are fibre volume fraction, fibre shape and fibre orientation. The other deals with siz ing industrial parts, which requires a good knowledge of the mechanical behaviour in a wide temperature range and under various mechanical loadings.

To answer the first question, a micro mechanical approach has been developed to model the extrac tion (or pullout) behaviour of metallic fibres from the refractory concrete $[30,31]$. The main parameters that are considered deal with geometric effects, test temperature effects and the thermal history of the composite. To answer the second one too, a macro mechanical approach models the thermomechanical behaviour [32].

The ceramic refractories considered are made of a geopolymer based matrix and cordierite aggregates. The matrix is obtained by mixing two commercial precursors [33]. The solid precursor consists of a major alumino silicate oxide and thermal silica fume. The liquid precursor is an aqueous solution of potassium polysilicate. Concerning aggregates, a mixture of four granulometric ranges of a cordierite grog is performed. The maximum aggregate size is close to $3 \mathrm{~mm}$. Metallic fibres are $0.38 \mathrm{~mm}$ in diameter, made of AISI 310 stainless steel and processed by cold drawing. The reinforced refractory concretes are shaped by mixing these different com ponents and by casting under vibrations. Complete polymerisation of the matrix is obtained after an isothermal heat treatment of $12 \mathrm{~h}$ at 80 or $110^{\circ} \mathrm{C}$, depending on the considered grade. The reinforced concrete is then heat treated at the operating tem perature or at a higher temperature in order to sta bilise the microstructure. The material composition and the described processing route are the results of previous studies of Cutard et al. [29, 34]. In the pre sent study, the use of forming tools made of rein forced ceramic refractory for the superplastic forming of aluminum alloys is the objective. In that case, the forming temperature is $500{ }^{\circ} \mathrm{C}$. As a consequence, samples were fired at $500{ }^{\circ} \mathrm{C}$ and mechanical tests performed at 20 or $500{ }^{\circ} \mathrm{C}$.

For the direct tensile test, a specific device has been developed in order to characterise the macroscopic mechanical behaviour of a ceramic refractory rein forced by steel fibres. Tests are performed on an MTS 810 servo hydraulic universal testing machine (www.mts.com). Specimens are glued on rigid water cooled metallic plates. Displacements and crack opening displacements are measured by two high temperature extensometers placed on two opposite faces of the specimen. Monotonic tests and cyclic loading/unloading tests are performed for each tem perature configuration with a displacement velocity of $0.04 \mathrm{~mm} \mathrm{~min}^{-1}$. At high temperature, the central part of the specimen is located in an MTS 653 resis tive furnace. The specimen geometry has been defined to allow tests from 20 to $500^{\circ} \mathrm{C}$. The sample size is of $150 \times 25 \times 25 \mathrm{~mm}^{3}$ and a $2 \mathrm{~mm}$ wide and $2 \mathrm{~mm}$ deep notch has been machined at the sample periphery in order to localise the crack initiation site in the extensometer gauge lengths and to allow accurate crack opening measurements. Transversal dimensions of samples are limited by the furnace size. Machining by grinding is performed on the two opposite faces that support the extensometers. A parallelism error lower than $0.05 \mathrm{~mm}$ is obtained between these two faces. A particular attention is also paid to ensure the better alignment between the sample axis and the machine loading axis in order to minimise bending loadings.

As described hereafter, 3D optical methods have been used:

1 To provide the validation step in the develop ment of links between the micro mechanical and the macro mechanical scales.

2 To measure displacement/strain fields during macroscopic tensile tests on notched samples.

\section{Measurement of the 3D Orientation of Metallic Fibres}

As an example, Figure 10A shows a tensile test sample of the ceramic refractory reinforced with metallic fibres. In Figure 10B the fibres which have been extracted from the refractory matrix at failure can be seen. The problem was to determine the $3 \mathrm{D}$ orienta tion of each fibre in order to analyse the sample behaviour through a single fibre pullout model. Stereovision has been used to achieve this.

The stereovision measurement is performed as follows:

1 The sample to be measured is placed in front of a stereovision sensor that has been calibrated beforehand (see section 'Calibration of the stere ovision sensor'). 

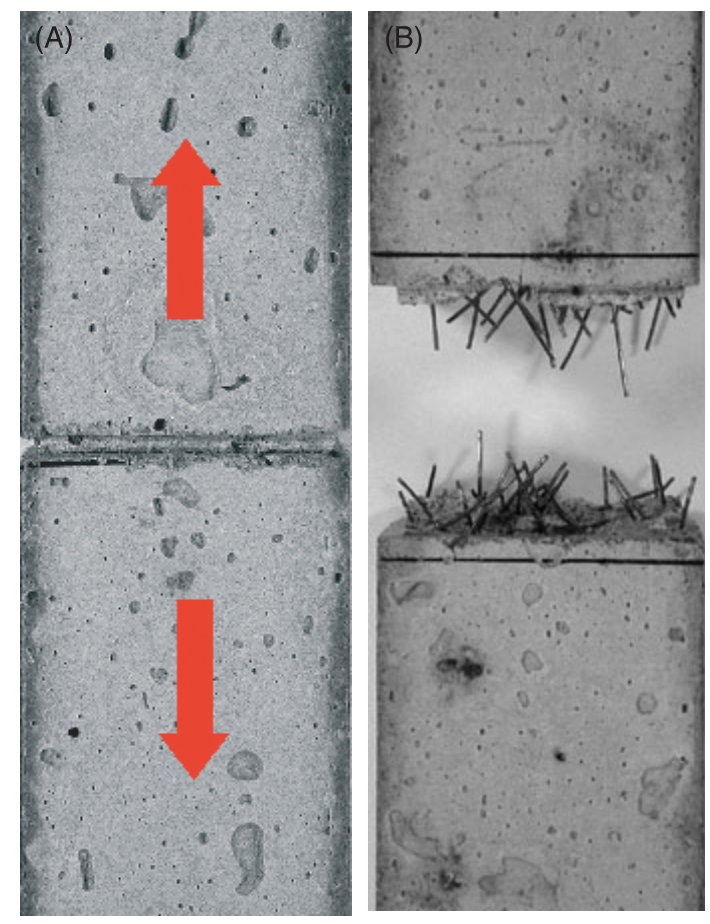

Figure 10: A sample of ceramic refractory reinforced with metallic fibres: (A) before the tensile test; (B) after rupture.

2 The operator selects one end of the fibre to be reconstructed in $3 \mathrm{D}$ in the left image (see Figure 11A). Using the epipolar constraint, the matching point in the right image is neces sary on the associated epipolar line (see Fig ure 11B).

3 The matched point is identified in the right image.

4 Operations 2 to 3 are repeated for the other end of the fibre currently under progress.

5 Operations 2 to 4 are repeated for all the fibres of the sample.
Figure 12 shows the 3D reconstruction of all the fibres of the sample. Using this technique, it has been possible to process a large number of samples (see some of them in Figure 13). Only a few minutes were required to process each sample.

The computation of the results is shown in the bar graph of Figure 14, showing the distribution of the fibre orientations. The fibre orientation corresponds to the angle value (in degree) between the fibre axis and the tensile loading direction, i.e. the direction parallel to the sample length.

The determination of such fibre orientation distri butions is helpful in many cases. First, they allow some statistical studies to be performed when con sidering the influence of varying some processing parameters on the 3D fibre distribution. Secondly, they are precious in the validation step of the pre diction of the macro mechanical behaviour from the micro mechanical pullout model. This analytical pullout model has been described in detail by Cail leux et al. [31] and is based on the strength material theory. Thus, at each step of the model, fibres are assumed to remain in their elastic domain. From a very limited number of tests, it allows the prediction of the pullout behaviour when varying parameters such as fibre diameter, fibre embedded length, incli nation angle, firing temperature and testing tem perature. As a consequence, the macro mechanical behaviour can be predicted when assuming that fibres act independently in the composite and when knowing both the fibre orientation distribution and the pullout curve for each fibre orientation angle.

Based on these assumptions, a macro mechanical model has been developed to describe the crack opening behaviour of such composites [30]. A valid ation of the model predictions has been made by
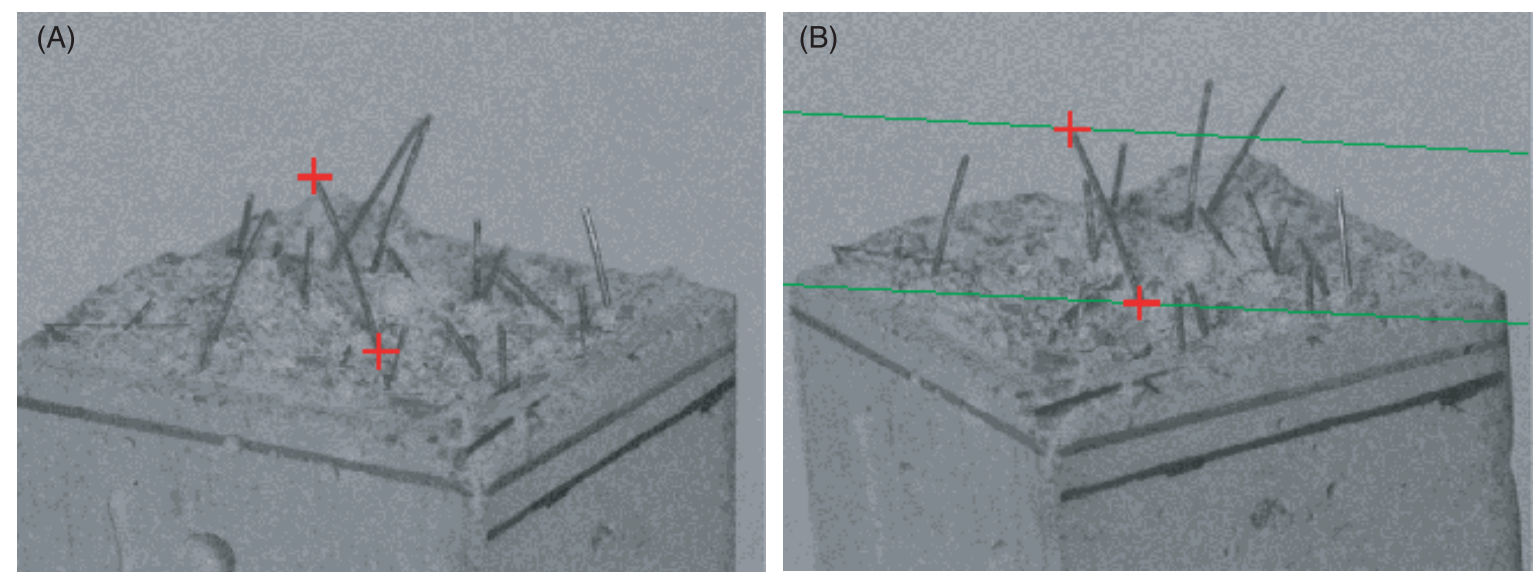

Figure I I: Pair of stereo-images: (A) ends of the fibre being processed; (B) associated epipolar lines that guide the search for correspondence 

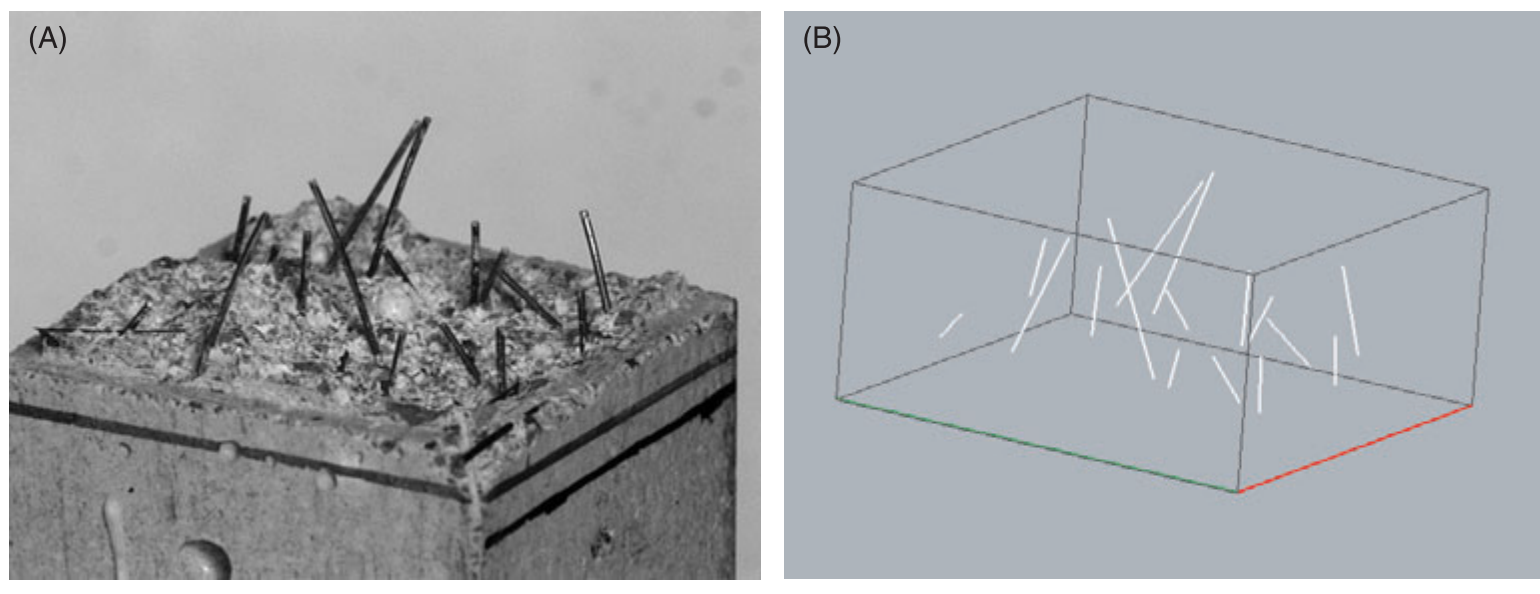

Figure 12: (A) Left image of the sample; (B) 3D reconstruction of all the fibres of the sample
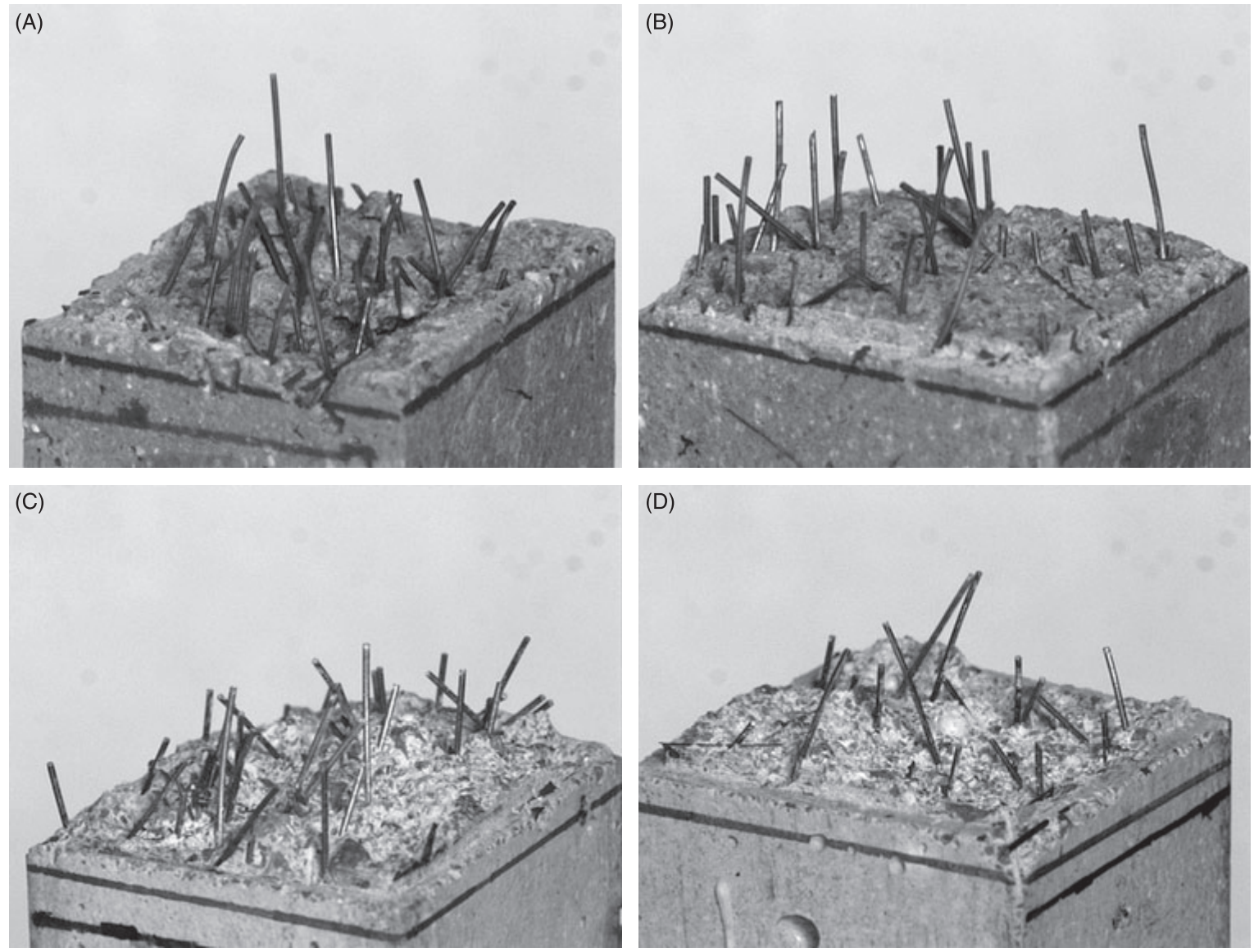

Figure 13: Some examples of samples that have been analysed using stereovision

comparing them with experimental results. Figure 15 shows the results of such a comparison in the case of $500^{\circ} \mathrm{C}$ tensile tests. As it is possible to determine the specific fibre distribution in the macrocrack of the tested specimens with 3D optical methods, their spe cific crack opening behaviour could be modelled. For each fibre orientation angle of the distribution, the pullout curve has been calculated by considering the single fibre pullout model. At each step of the macro mechanical model, a displacement increase is performed and the corresponding load value is calcu lated from a weighted summation of the load values of each pullout curve. For each orientation angle, the weight corresponds to the number of fibres that has been determined from stereovision. As a consequence, the comparison between experimental and model 


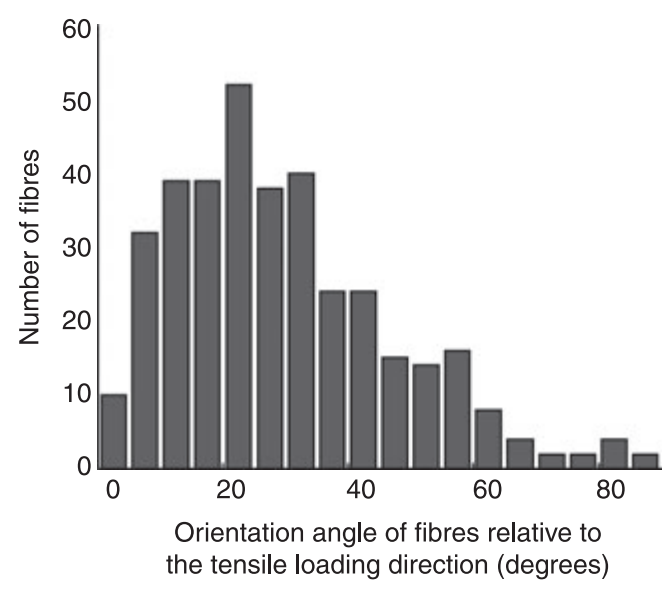

Figure 14: Distribution of fibre orientations

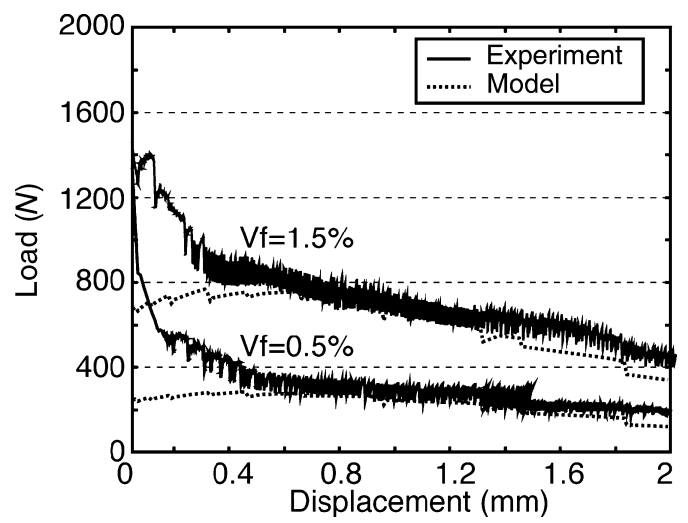

Figure 15: Crack opening during $500^{\circ} \mathrm{C}$ tensile tests on a fibre reinforced ceramic refractory: comparison between modelling and experiment, for two fibre volumic fractions (Vf)

results can be made on the exact microstructures that have been tested. A good agreement is observed be tween experiments and model predictions for crack opening displacements higher than $0.4 \mathrm{~mm}$. For lower crack opening values, the large discrepancy between experimental data and the model is mainly due to the geometrical hypothesis of the actual pullout model (see [31] for more details). Therefore, this approach allows the prediction of the macro mechanical behaviour of fibre reinforced ceramic refractories. These qualitative and quantitative indi cations are helpful in optimising the composite formulation and design.

Experiments show that non zero strain levels are measured during the progressive crack opening of the tensile test specimens. Such a behaviour has a major influence in limiting the crack propagation and in allowing stress redistribution and multi cracking in the case of parts subjected to complex loadings [35].

\section{D Displacement Field Measurement}

The stereo correlation technique discussed in the section '3D Displacement Measurement' has been used during a tensile test to measure the $3 \mathrm{D}$ dis placement/strain field on the surface of a ceramic refractory sample reinforced with metallic fibres.

A notched sample (see Figure 10) was subjected to a tensile test, carried out on an electromechanical universal test machine. For reasons of comparison, the sample was equipped with two mechanical extensometers placed on two opposite faces of the sample (see Figure 16). During the test, a sequence of stereo images was acquired, for which the displace ment value measured by the mechanical extensom eters was recorded. Using the combined tracking and stereo correlation technique described in the section '3D Displacement Measurement', the 3D displace ment of several pairs of points (about 100) distributed along the crack was measured. These pairs of points
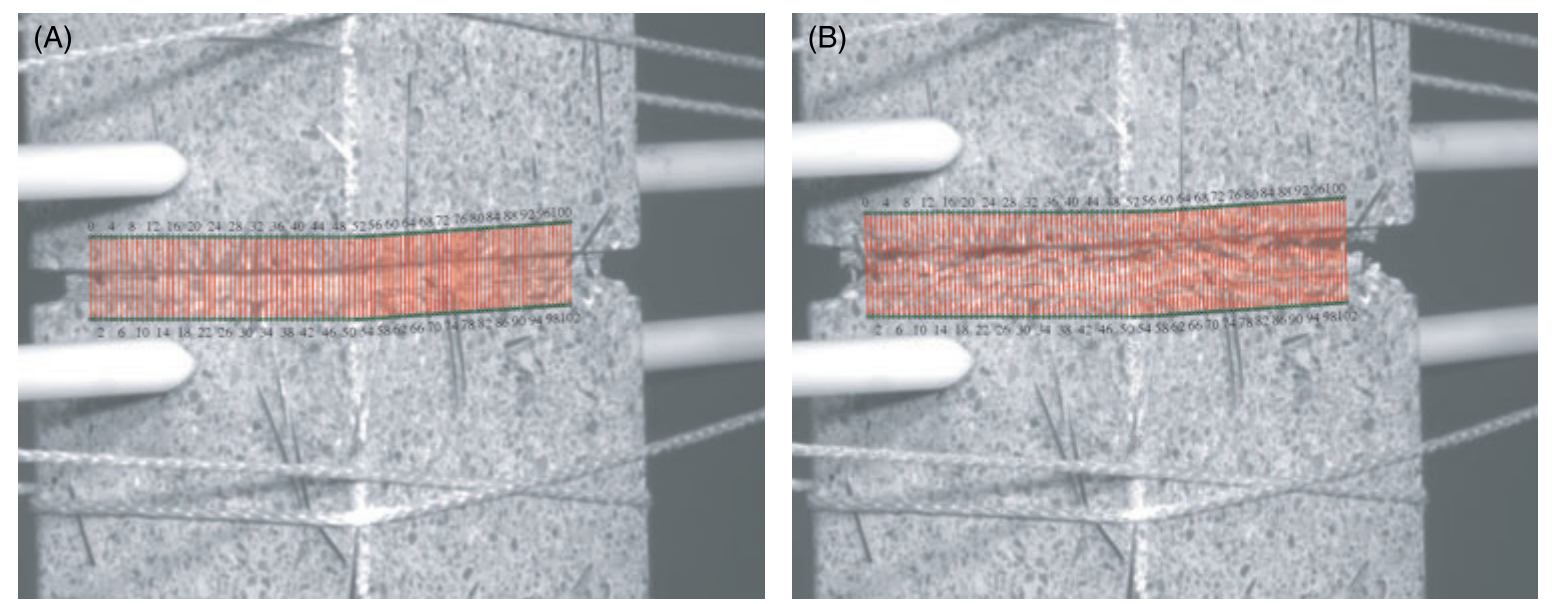

Figure 16: Virtual and mechanical (white stems) extensometers: (A) at the beginning of the test; (B) at the end of the test. Note that this figure presents lightened images in order to emphasise the location of the virtual extensometers 


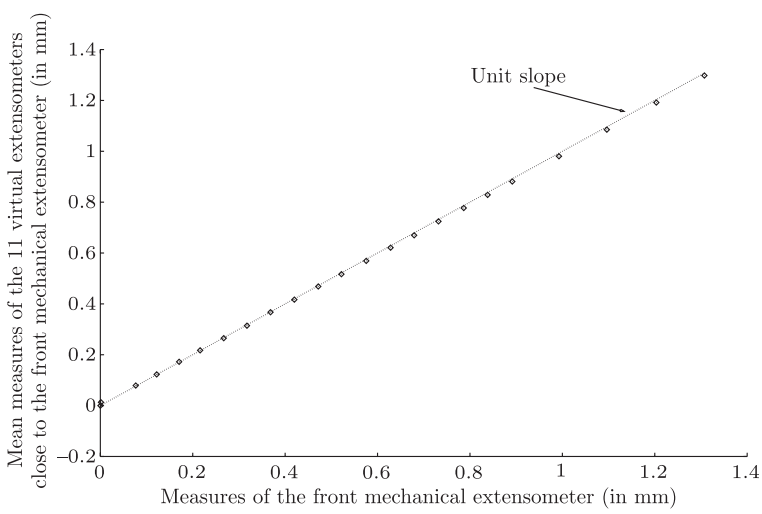

Figure 17: Displacements measured mechanically and by stereo-correlation

can be regarded as virtual extensometers. Figure 16 shows the pairs of points in the first left image and the last left image of the sequence.

Figure 17 shows the very good agreement between the displacements measured by the mechanical extensometer placed on the front face of the sample and the displacements computed using the virtual extensometers (modulus of the 3D displacement). The 3D virtual extensometers provided by the ste reo correlation technique are very useful for two reasons:

- They provide 3D measurements (direction and amplitude of the displacement) without any contact with the sample.

- They are placed in the images at the processing level: the number of extensometers and their position can be chosen interactively (only one experiment is carried out).

Therefore the virtual extensometers bypass the main drawbacks of mechanical extensometers, which are:

- They provide only $1 \mathrm{D}$ information (length vari ation).

- Some problems can arise because of a bad mechanical contact with the sample.

- The measurements are difficult to analyse if strain localisation phenomena occur close to the contact points.

- The experiment must be repeated if one of the two previous points occurs.

Figure 18 provides the displacement measured by each virtual extensometer at different image acqui sition times. For a given curve, the measures show some fluctuations. This can be explained by the fact that some virtual extensometers have been placed on some microstructural heterogeneities of the material (aggregates, fibres, etc.) that are present on the sam ple surface.
In Figure 18, the virtual extensometers placed on the front face of the sample should be distinguished from those placed on the lateral face of the sample ${ }^{3}$ (see Figure 16). Concerning the front face of the sample, the mechanical extensometer corresponds to the virtual extensometers numbered 10 to 20 .

If the displacement measurements on the front face are compared with those on the lateral face of the sample, it can be observed that:

- For a given curve (corresponding to a given acquisition time), the displacements increase from the left side to the right side of the front face.

- For a given curve, the displacements increase from the front to the bottom of the lateral face.

These results show that a parasitic bending compo nent was introduced in the tensile sample. This can be due to a residual misalignment between the sam ple axis and the loading axis of the universal testing machine, even if precautions are taken during the gluing step of the sample, or to a non symmetric crack propagation, as regularly observed during such tensile tests. The 3D optical measurement allows this bending component to be detected and quantified, which is not possible with only two mechanical extensometers.

\section{Conclusions}

An accurate new stereovision technique has been developed for measuring the 3D shape of a static object or the 3D displacement/strain field of an object being deformed. The stereovision technique, which provides 3D measurements, appears helpful in the study of fibre reinforced ceramic refractories. The 3D measurements of the fibre orientation have been used in the validation step of the prediction of the high temperature crack opening behaviour from the results of a pullout model. These 3D op tical methods gave the possibility to determine the specific fibre distribution in the macrocrack of the tested specimens. As a consequence, their specific crack opening behaviour could be modelled and compared with the experimental results. The determination of such fibre orientation distribu tions will be useful to study the links between the microstructure of such composites and their pro cessing route. As fibre orientations and distributions are critical in these materials, the ability to quantify the influence of varying processing parameters on

\footnotetext{
${ }^{3}$ In Figure 16, the 'lateral face of the sample' corresponds to the face without mechanical extensometer.
} 


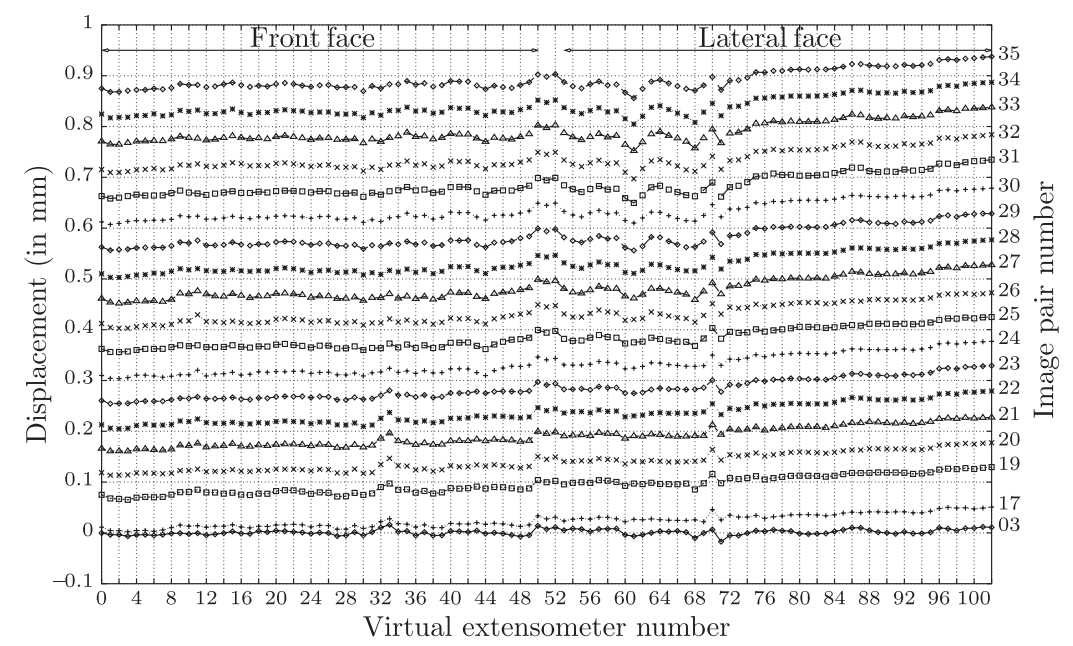

Figure 18: Displacements measured by each virtual extensometer at different image acquisition times

fibre distribution remains a priority. This study has shown this is possible with stereovision based methods.

The measurement of displacement/strain fields ob tained from the stereovision technique provides 3D information that permits the study of the mechanical behaviour of refractory composites. The technique appears to be well suited to characterise the crack opening (or post peak) behaviour at room tempera ture. Compared to classical measurements with mechanical extensometers, stereovision allows dis placement/strain fields on the sample surface to be obtained, avoiding the experimental difficulties due to contact rupture between a mechanical extensometer and the sample or to strain localisation phenomena. Another important point is the possibility of analysing surface strain fields to obtain behaviour curves under non perfectly homogeneous stress or strain fields.

In this paper two challenges have been identified. One deals with using the stereovision method to characterise the pre peak behaviour of refractory composites, where strain levels as low as $10^{-4}$ have been measured. The other one deals with performing such measurements with a high accuracy at high temperature. The authors are currently working on these challenging issues.

\section{REFERENCES}

1. Sutton, M. A., McNeill, S. R., Helm, J. D. and Schreier, H. W. (2000) Computer vision applied to shape and defor mation measurement. In: Trends in Optical Nondestructive Testing and Inspection (P. K. Rastogi and D. Inaudi, Ed.), Elsevier Science, Oxford, 571589.

2. Sutton, M. A., McNeill, S. R., Helm, J. D. and Chao, Y. J. (2000) Advances in two dimensional and three dimen sional computer vision. In: Photomechanics, Topics in Applied Physics, Vol. 77. (P. K. Rastogi Ed.), Springer Ver lag, London, 323372.
3. Galanulis, K. and Hofmann, A. (1999) Determination of forming limit diagrams using an optical measurement system. 7th Int. Conf. Sheet Metal SheMet'99, 2728 September, Erlangen, Germany.

4. Kahn Jetter, Z. L. and Chu, T. C. (1990) Three dimen sional displacement measurements using digital image correlation and photogrammic analysis. Exp. Mech. 30, 1016.

5. Langerak, N., Atzema, E. and Essing, J. (2000) Strain measurements with the PHAST system. Int. Deep Drawing Research Group (IDDRG'2000) Meeting of the Working Groups, 89 June, Ann Arbor, MI, USA.

6. Luo, P. F., Chao, Y. J., Sutton, M. A. and Peters, W. H. (1993) Accurate measurement of three dimensional defo rmable and rigid bodies using computer vision. Exp. Mech. 33, 123132 .

7. Orteu, J. J., Garric, V. and Devy, M. (1997) Camera calib ration for 3D reconstruction: application to the measure of 3D deformations on sheet metal parts. Eur. Symp. Lasers, Optics and Vision in Manufacturing, 1620 June, Munich, Germany.

8. Synnergren, P. and Sjödahl, M. (1999) A stereosco pic digital speckle photography system for $3 \mathrm{D}$ dis placement field measurements. Optics Lasers Eng. 31, 425443.

9. Vogel, J. H. and Lee, D. (1989) An automated two view method for determining strain distributions on deformed surfaces. J. Mater. Shaping Technol. 6, 205216.

10. Robert, L., Buffa, M. and Hebert, M. (1995) Weakly calib rated stereo perception for rover navigation. Fifth Int. Conf. Computer Vision (ICCV'95), 2023 June, Cambridge, MA, pp. 4651.

11. Garcia, D., Orteu, J. J. and Devy, M. (2000) Accurate cal ibration of a stereovision sensor: comparison of different approaches. Vision, Modeling, and Visualization 2000, 22 24 November 2000, Saarbrücken, Germany.

12. Garcia, D. (2001) Mesure de formes et de champs de déplacements tridimensionnels par stéréo corrélation d'images. PhD thesis (in French), Institut National Poly technique de Toulouse, France. 
13. Claudinon, S., Lamesle, P., Orteu, J. J. and Fortunier, R. (2000) Monitoring distortions of metallic parts during heat treatment. 5th ASM Heat Treatment and Surface Eng. Conf. Eur., 79 June, Gothenburg, Sweden.

14. Garcia, D. and Orteu, J. J. (1999) 3D deformation meas urement using stereo correlation applied to the forming of metal or elastomer sheets. Int. Workshop Video Controlled Materials Testing and In Situ Microstructural Characterization, 1618 November, Nancy, France.

15. Garcia, D. and Orteu, J. J. (2001) 3D deformation meas urement using stereo correlation applied to experimental mechanics, 10th FIG Int. Symp. Deformation Measurements, 1922 March, Orange, CA.

16. Garcia, D., Orteu, J. J. and Penazzi, L. (2002) A combined temporal tracking and stereo correlation technique for accurate measurement of 3D displacements: application to sheet metal forming. J. Mater. Process. Technol. 1, 125 126, 736 742, Elsevier Science, September 2002.

17. Salvi, J., Armangué, X. and Battle, J. (2002) A comparative review of camera calibrating methods with accuracy evaluation. Pattern Recognition 35, 16171635.

18. Triggs, B., McLauchlan, P., Hartley, R. and Fitzgibbon, A. (2000) Bundle adjustment a modern synthesis. In: Vision Algorithms: Theory and Practice (B. Triggs, A. Zisserman and R. Szeliski, Eds). Springer Verlag, London, 298372.

19. Ayache, N. and Hansen, C. (1988) Rectification of images for binocular and trinocular stereovision. 9th IEEE Int. Conf. Pattern Recognition, November 1988, Rome, Italy, pp. 1116.

20. Loop, C. and Zhang, Z. (1999) Computing rectifying homographies for stereo vision. Microsoft Research Technical Report MSR TR 99 21, April 1999.

21. Faugeras, O. D. (1993) Three Dimensional Computer Vision: A Geometric Viewpoint. The MIT Press, Cambridge, MA.

22. Sutton, M. A., Wolters, W. J., Peters, W. H., Ranson, W. F. and McNeill, S. R. (1983) Determination of displacements using an improved digital correlation method. Image Vision Comput. 1, 133139.

23. Hartley, R. I. and Sturm, P. (1997) Triangulation. Computer Vision and Image Understanding (CVIU'97) 68, 146157.
24. Cornille, N. (2005) Accurate 3D Shape and Displacement Measurement using a Scanning Electron Microscope. PhD thesis, INSA Toulouse (France) and University of South Carolina (Columbia, SC).

25. Allais, L., Bornert, M., Bretheau, T. and Caldemaison, D. (1994) Experimental characterization of the local strain field in a heterogeneous elastoplastic material. Acta Metal. Mater.. 42, 38653880.

26. Routschka, G. (1997) Refractory materials. Vulkan Verlag, Essen.

27. Lemaistre, H. (1998) Étude des propriétés thermoméca niques de divers réfractaires. PhD thesis (in French). INSA, Lyon.

28. Shah, S. P. and Ouyang, C. (1991) Mechanical behavior of fibre reinforced cement based composites. J. Am. Ceramic Soc. 74, 27272953.

29. Cutard, T., Cailleux, E. and Bernhart, G. (2002) Pullout of metallic fibres from a refractory concrete matrix. Composites Part A. 33, 14611466.

30. Cailleux, E. (2001) Microstructure et comportement thermomécanique d'un béton réfractaire renforcé par des fibres métalliques. PhD thesis (in French), École des Mines de Paris.

31. Cailleux, E., Cutard, T. and Bernhart, G. (2005) Pullout of steel fibres from a refractory castable: experiment and modelling. Mech. Mater. 37, 427445.

32. Cutard, T., Cailleux, E., Nazaret, F. and Bernhart, G. (2004) Metallic fibre reinforced refractory concretes. In: Advances in Refractories for the Metallurgical Industries IV, Com2004 (M. Rigaud and C. Allaire, Eds), Hamilton, Canada: 296307.

33. Davidovits, J. (1994) Geopolymer inorganic polymeric new materials. J. Mater. Educ. 16, 91139.

34. Cutard, T., Cailleux, E., Lours, P. and Bernhart, G. (1999) Structural and mechanical properties of a refractory con crete for superplastic forming tools. Industrial Ceramics 19, 100102.

35. Bernhart, G., Nazaret, F. and Cutard, T. (2007) Fibre reinforced refractory castables: an alternative solution for SPF die manufacturing. Mater. Sci. Forum 551 552, 3742. 